\title{
Genetic profile of adenoid cystic carcinomas (ACC) with high-grade transformation versus solid type
}

\author{
Ana Flávia Costa ${ }^{\mathrm{a}, *}$, Albina Altemani ${ }^{\mathrm{a}}$, Hedy Vékony ${ }^{\mathrm{b}}$, Elisabeth Bloemena ${ }^{\mathrm{b}}$, Florentino Fresno ${ }^{\mathrm{c}}$, \\ Carlos Suárez ${ }^{\mathrm{d}}$, José Luis Llorente ${ }^{\mathrm{d}}$ and Mario Hermsen ${ }^{\mathrm{d}}$ \\ ${ }^{a}$ Department of Pathology, University of Campinas/UNICAMP, Campinas, Brazil \\ ${ }^{\mathrm{b}}$ Department of Pathology, VU University Medical Center, Amsterdam, The Netherlands \\ ${ }^{\mathrm{c}}$ Department of Pathology, IUOPA, Hospital Universitario Central de Asturias, Oviedo, Spain \\ ${ }^{\mathrm{d}}$ Department of Otolaryngology, IUOPA, Hospital Universitario Central de Asturias, Oviedo, Spain
}

\begin{abstract}
Background: ACC can occasionally undergo dedifferentiation also referred to as high-grade transformation (ACCHGT). However, ACC-HGT can also undergo transformation to adenocarcinomas which are not poorly differentiated. ACC-HGT is generally considered to be an aggressive variant of ACC, even more than solid ACC. This study was aimed to describe the genetic changes of ACC-HGT in relation to clinico-pathological features and to compare results to solid ACC.

Methods: Genome-wide DNA copy number changes were analyzed by microarray CGH in ACC-HGT, 4 with transformation into moderately differentiated adenocarcinoma (MDA) and two into poorly differentiated carcinoma (PDC), 5 solid ACC. In addition, Ki-67 index and p53 immunopositivity was assessed.

Results: ACC-HGT carried fewer copy number changes compared to solid ACC. Two ACC-HGT cases harboured a breakpoint at 6q23, near the cMYB oncogene. The complexity of the genomic profile concurred with the clinical course of the patient. Among the ACC-HGT, p53 positivity significantly increased from the conventional to the transformed (both MDA and PDC) component.

Conclusion: ACC-HGT may not necessarily reflect a more advanced stage of tumor progression, but rather a transformation to another histological form in which the poorly differentiated forms (PDC) presents a genetic complexity similar to the solid ACC. Keywords: Adenoid cystic carcinoma, high-grade transformation, dedifferentiation, microarray CGH
\end{abstract}

\section{Introduction}

Adenoid cystic carcinoma is a slow-growing tumor presenting a dual cellular composition, i.e., ductal (luminal) and myoepithelial cell differentiation and three major growth patterns: tubular, cribriform and solid [6]. The solid growth pattern has been considered to be an adverse prognosticator [5,21,31] and in a three-tired system for grading ACC, tumors having more than $30 \%$ of the solid component are classified as grade III or poorly differentiated. Grade I tumors are those with tubular and cribriform areas but without solid com-

\footnotetext{
*Corresponding author: Ana Flávia Costa, Departamento de Anatomia Patológica, Faculdade de Ciências Médicas, UNICAMP, Rua Tessália Vieira de Camargo 126, 13084-971 Campinas, São Paulo, Brazil. Tel.: +55 19 32893897; E-mail: costa.anaflavia@ gmail.com.
}

ponents whereas grade II are pure or mixed cribriform with less than $30 \%$ of solid areas [31]. Grade III ACC has been associated with increased disease mortality and greater frequency of aneuploidy than grade I or II tumors [8]. Furthermore, comparing low-grade foci with high-grade ones within the same tumor revealed a greater number of mutations at either the p53 or $\mathrm{Rb}$ genes in the latter [19].

ACC can occasionally undergo transformation into poorly differentiated adenocarcinoma or undifferentiated carcinoma. This phenomenon has been referred to as dedifferentiation or high-grade transformation (ACC-HGT) and there have been only 33 reported cases so far $[1,3,4,10,12,15-17,25,26]$. This process was first believed to occur in low-grade ACCs without morphological recognizable changes, as an abrupt transition, but recently cases have been described 
showing a gradual transformation of solid ACC into high-grade adenocarcinoma $[25,26]$.

ACC-HGT is generally considered to be an aggressive variant of ACC, even more than solid ACC [26]. However, recently it has been claimed that high-grade transformation of ACC may, in addition to poorly differentiated carcinomas, also result in adenocarcinomas with a moderate differentiation [1].

The pathogenesis of high-grade transformation of ACC is poorly understood, partly because few studies have been dedicated to this tumor type. Some molecular studies reported TP53 mutations, loss of heterozygosity at the TP53 locus $[3,17]$ and strong overexpression of p53 protein in high-grade components $[3,4,17$, 26], suggesting that p53 alterations may play a significant role in the pathogenesis of high-grade transformation of ACC [3].

Genetic characterization of these lesions may give more insight in this complex matter. This study was aimed to compare the ACC-HGT and solid type ACC using genome-wide high-resolution microarray $\mathrm{CGH}$ analysis. In addition, the genetic changes were correlated with clinical outcome.

\section{Material and methods}

\subsection{Material}

The present study included 11 paraffin-embedded carcinoma samples of patients with ACC diagnosed between 1996 and 2007: 6 cases ACC-HGT and 5 cases solid ACC. Five cases of ACC-HGT were obtained from the archives of the Department of Pathology of the University of Campinas, Brazil, 1 case of ACC-HGT of the Department of Pathology of the Hospital Universitario Central de Asturias, Spain and 5 cases of solid ACC of the Department of Pathology, VU University Medical Center, The Netherlands. The solid ACC have previously been described as part of a larger series [32]. Hematoxylin-eosin (H\&E) stained slides from each tissue block were reviewed (Fig. 1) to confirm the pathological diagnosis. The transformed

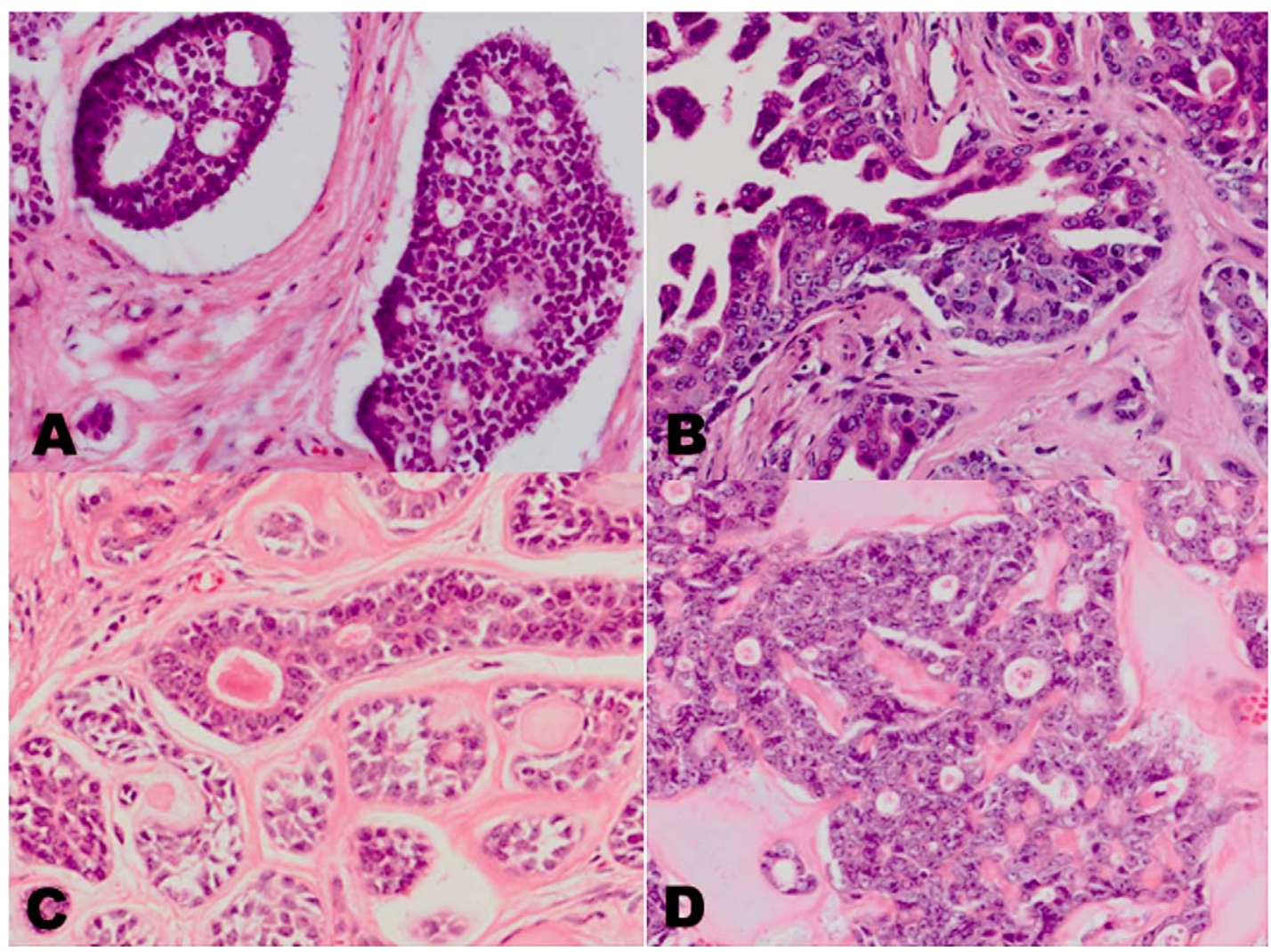

Fig. 1. ACC-HGT to a moderately differentiated adenocarcinoma, case C (A, B). ACC-HGT to poorly differentiated carcinoma, case F (C, D). $\mathrm{H} \& \mathrm{E}$ original magnification $200 \times(\mathrm{A}, \mathrm{B}, \mathrm{C}$ and D). (Colors are visible in the online version of the article; http://dx.doi.org/10.3233/ACP-CLO2010-0547.) 
component was identified according to the criteria described by Seethala et al. [26] and all cases showed the following features: proliferation of tumor cells with at least a focal loss of myoepithelial cells surrounding tumor nests, nuclear size at least 2-3 times the size of tubular/cribriform ACC nuclei, thickened irregular nuclear membranes and prominent nucleoli in a majority of cells. In addition (Table 1), based on the degree of gland formation (differentiation), cellular pleomorphism and mitotic activity, the transformed components were classified into: moderately differentiated adenocarcinomas (MDA) when at least 2/3 exhibited gland differentiation and poorly differentiated carcinomas (PDC) those with scarce or absent gland differentiation [34]. The conventional component was classified in a three-tired system proposed by Szanto et al. [31], which is widely used in the literature.

The ACC-HGT group consisted of 1 male and 5 female patients ranging from 44 to 65 years of age, with a median of 56 years. Two tumors occurred in the submandibular gland, 2 in paranasal sinus and 1 in palate and 1 in parotid gland. Four cases underwent transformation into MDA and two into PDC. The solid ACC group comprised of 2 male and 3 female patients ranging from 33 to 66 years of age, with a median of 52 years. Three tumors occurred in the parotid gland and 1 in the submandibular gland. The exact origin of case 2 could not be determined; it concerned a large mass located in the oropharynx and nasopharynx. The follow-up time was 7-144 months (mean 55 months) for the ACC-HGT and 7-81 months (mean 43 months) for the solid ACC. The clinical and pathologic data of all cases are summarized in Table 1 .

\subsection{Microdissection and DNA extraction}

Tumor tissue of 5 solid ACC and 6 ACC-HGT was obtained from 10 paraffin sections of $10 \mu \mathrm{m}$. Regions of interest (conventional and transformed areas) of the tumors were carefully dissected manually, on the basis of H\&E-stained slides. Tumor DNA was extracted using Qiagen extraction kits (Qiagen $\mathrm{GmbH}$, Hilden, Germany) according to the manufacturer's recommendations. Special care was taken to obtain high quality DNA from the formaldehyde-fixed, paraffinembedded tissues. DNA extracted from archival material can be partly degraded and cross-linked, the extent of which depends on the $\mathrm{pH}$ of the formaldehyde and the time of the fixation before paraffin embedding. To improve the quality of the isolated DNA, we have applied an elaborate extraction protocol es- pecially for paraffin tissues, which includes thorough deparaffination with xylene, methanol washings to remove all traces of the xylene and 24-h incubation in $1 \mathrm{~mol} / \mathrm{l}$ sodium thiocyanate to reduce cross-links. Subsequently, the tissue pellet is dried and digested for 3 days in lysis buffer with high doses of proteinase $\mathrm{K}$ (final concentration $2 \mu \mathrm{g} / \mu \mathrm{l}$, freshly added twice a day). Finally, the DNA was purified with Qiagen columns (QIAamp DNA mini-kit Qiagen GmbH, Hilden, Germany). With this protocol, most formaldehyde-fixed, paraffin-embedded tissue samples yielded DNA of relatively good quality, with A260/A280 values between 1.7 and 2.0 measured by Nanodrop (Thermo Scientific, Wilmington, DE, USA) and lengths between 2000 and 20,000 bp. Before performing microarray $\mathrm{CGH}$, we performed an additional quality test using the ENZO Bioscore Screening and Amplification kit (Enzo Life Sciences, Lörrach, Germany). The assay consists of an isothermal whole genome amplification reaction using $100 \mathrm{ng}$ of DNA, followed by a purification by QIAquick PCR Purification columns (Qiagen GmbH, Hilden, Germany) and measurement of the DNA concentration by Nanodrop (Thermo Scientific, Wilmington, DE, USA). Only those samples that gave a total yield of 3.0 or more were used for microarray CGH analysis.

\subsection{Microarray $\mathrm{CGH}$}

Microarray CGH analysis was performed as described previously by Buffart et al. [2]. Briefly, sample DNA and reference DNA (extracted and pooled from blood of 18 different healthy female donors) were differently labeled by using the Enzo Genomic DNA Labeling kit according to the manufacturer's instructions (Enzo Life Sciences, Lörrach, Germany). Five hundred nanograms test and $500 \mathrm{ng}$ pooled reference DNA were hybridized to a $180 \mathrm{k}$ oligonucleotide array (SurePrint G3 Human CGH Microarray Kit $4 \times 180 \mathrm{~K}$, Agilent Technologies, Palo Alto, CA, USA). Hybridization and washing took place in a specialized hybridization chamber (Agilent Technologies). Images were acquired using a Microarray scanner G2505B (Agilent Technologies, Amstelveen, The Netherlands). Analysis and data extraction were quantified using feature extraction software (version 9.1, Agilent Technologies). Normalization of the calculated ratios was done against the mode of the ratios of all autosomes. Graphics were plotted using a moving average of $\log 2$ ratios of 5 neighboring clones. Gains and losses were defined as deviations of 0.2 or more 
Table 1

Clinicopathologic parameters

\begin{tabular}{|c|c|c|c|c|c|c|c|c|c|c|c|c|c|}
\hline \multirow[t]{2}{*}{ Case } & \multirow[t]{2}{*}{ Age } & \multirow[t]{2}{*}{ Sex } & \multirow[t]{2}{*}{ Site } & \multirow[t]{2}{*}{$\mathrm{T}$} & \multirow[t]{2}{*}{ Treatment } & \multirow[t]{2}{*}{$\begin{array}{l}\text { Local } \\
\text { recurrence }\end{array}$} & \multirow[t]{2}{*}{$\begin{array}{c}\text { Distant } \\
\text { metastasis }\end{array}$} & \multirow[t]{2}{*}{$\begin{array}{l}\text { Follow-up } \\
\text { (mo) }\end{array}$} & \multirow{2}{*}{$\begin{array}{c}\text { Time } \\
\text { disease } \\
\text { free }(\mathrm{mo})\end{array}$} & \multirow[t]{2}{*}{ Outcome } & \multicolumn{2}{|c|}{$\begin{array}{l}\text { Histological } \\
\text { features }\end{array}$} & \multirow{2}{*}{$\begin{array}{c}\text { Glandular } \\
\text { differen- } \\
\text { tiation }\end{array}$} \\
\hline & & & & & & & & & & & $\mathrm{CA}$ & TA & \\
\hline \multicolumn{14}{|c|}{ ACC with transformation } \\
\hline A & 44 & $\mathrm{~F}$ & Submandibular & $\mathrm{T} 2$ & $\mathrm{SE}+\mathrm{RT}$ & No & No & 18 & 18 & NA & $\mathrm{T} / \mathrm{C}$ & MDA & Frequent \\
\hline B & 55 & $\mathrm{~F}$ & Palate & $\mathrm{T} 4$ & $\mathrm{SE}+\mathrm{RT}$ & No & No & 140 & 132 & NED & $\mathrm{S}$ & MDA & Frequent \\
\hline $\mathrm{C}$ & 65 & M & Paranasal sinus & $\mathrm{T} 4$ & $\mathrm{SE}+\mathrm{RT}$ & No & No & 8 & 8 & Dead & S & MDA & Frequent \\
\hline $\mathrm{D}$ & 49 & $\mathrm{~F}$ & Parotid & $\mathrm{T} 3$ & $\mathrm{SE}+\mathrm{RT}$ & No & Liver & 33 & 19 & Alive & S & MDA & Frequent \\
\hline $\mathrm{E}$ & 61 & F & Paranasal sinus & $\mathrm{T} 2$ & $\mathrm{SE}+\mathrm{RT}$ & Yes & No & 144 & 132 & Alive & S & PDC & Scarce \\
\hline $\mathrm{F}$ & 64 & F & Submandibular & $\mathrm{T} 2$ & $\mathrm{SE}+\mathrm{RT}$ & No & Liver & 7 & 5 & DOD & S & PDC & Scarce \\
\hline \multicolumn{14}{|c|}{ Solid ACC } \\
\hline 1 & 46 & M & Submandibular & $\mathrm{T} 1$ & SE & No & $\begin{array}{l}\text { Liver, lung, } \\
\text { bone }\end{array}$ & 7 & 2 & DOD & S & - & - \\
\hline 2 & 58 & F & $\begin{array}{l}\text { Oropharynx/ } \\
\text { Nasopharynx }\end{array}$ & NA & $\mathrm{SE}+\mathrm{RT}$ & Yes & No & 64 & 46 & DOD & S & - & - \\
\hline 3 & 57 & M & Parotid & NA & $\mathrm{SE}+\mathrm{RT}$ & Yes & Liver & 10 & 10 & Alive & S & - & - \\
\hline 4 & 66 & $\mathrm{~F}$ & Parotid & $\mathrm{T} 2$ & $\mathrm{SE}+\mathrm{RT}$ & Yes & No & 81 & 56 & DOD & S & - & - \\
\hline 5 & 33 & $\mathrm{~F}$ & Parotid & $\mathrm{T} 2$ & $\mathrm{SE}+\mathrm{RT}$ & No & No & 51 & 51 & Alive & $S$ & - & - \\
\hline
\end{tabular}

differentiated carcinomas; RT - radiotherapy; S - solid; SE - surgical excision; T - TNM classification; TA - transformed area; T/C - tubular/cribriform. 
from $\log 2$ ratio $=0.0$. High-level amplification was considered when at least 2 neighboring clones reached a $\log 2$ ratio of 1.0 or higher. The locations of possible copy number variations (rather than copy number alterations) were verified with the database of genomic variants and mapped according the human genome build NCBI 36 (http://projects.tcag.ca/variation/). The microarray CGH analyses of the 5 solid ACC have been performed as part of a previous study, using a home-made microarray consisting of approximately 4500 BAC-PAC clones [32].

\subsection{Immunohistochemistry}

One paraffin block from each case was chosen for the immunohistochemical study and the following antibodies (DAKO, Carpenteria, CA, USA) were used: Ki-67 (MIB1 - dilution 1:150), p53 (DO-7 - dilution $1: 100)$ and alpha smooth muscle actin $(\alpha$-SMA, 1A4-dilution 1:200). The $5 \mu \mathrm{m}$ sections were deparaffinized, hydrated and endogenous peroxidase activity was quenched by immersion of the slides in 3\% hydrogen peroxide. Antigen retrieval (AR) was performed for Ki-67 and p53 by using Tris-EDTA (pH 9.0), heating 5 min in a pressure cooker. For $\alpha$-SMA, AR was not done. Staining was done at room temperature on an automatic staining workstation (Autostainer; DAKO, Carpenteria, CA, USA). Subsequently, for all antibodies the sections were incubated with the primary antibody, and afterwards with the Envision peroxidase system (Envision Plus; DAKO, Carpenteria, CA, USA) and with 3,3'-diaminobenzidine tetrahydrochloride (DAB) chromogen used as the substrate (DAKO). Counterstaining with hematoxylin for $1 \mathrm{~min}$ was the final step. After staining, the slides were dehydrated through graded alcohols and mounted with a coverslip. Negative controls were run by omitting primary antibodies.

Immunostaining of alpha smooth muscle actin $(\alpha$-SMA) and p63, the latter as part of a previous study [1], was done in all cases of ACC-HGT for detection of myoepithelial cells contributing the selection of the transformed area for DNA extraction. In Ki-67 and p53-stained sections, three hotspot areas were chosen for counting of positive cells at $40 \times$ magnification. To quantify positive and negative cells, images were obtained from three areas and analyzed with Imagelab analysis software (version 2.4, Softium informática LTDA-ME, São Paulo, Brazil). Ki-67 and p53 indexes were calculated as the percentage of positive cells in relation to all tumor cells in these three areas in each sample.

\subsection{Statistical analysis}

Possible correlations between genetic and clinicopathological parameters were statistically analyzed by SPSS 12.0 software for Windows (SPSS ${ }^{\circledR}$ Inc., IL, USA), using the Fisher exact $\chi^{2}$ test and Student's $t$-test. Kaplan-Meier analysis was performed for estimation of survival, comparing distributions of survival through the logarithmic range test (log-rank test). $p$-Values below 0.05 were considered significant.

\section{Results}

\subsection{Clinical follow-up}

All patients except the case 1 of solid ACC underwent radiotherapy after resection. Chemotherapy was not used in any case. During the follow-up period, 3 of 6 ACC-HGT and 4 of 5 solid ACC developed either a recurrence or a metastasis. The overall survival (Fig. 3(a)) of the 6 ACC-HGT was more favorable than the 5 solid ACC (mean 58 versus 42 months) and this was the same with regard to the disease-free survival (mean 52 versus 33 months), although this did not reach statistical significance (Fig. 3(b)).

\subsection{Microarray $C G H$}

Two of 6 ACC-HGT yielded a bad quality of DNA and had to be excluded from microarray CGH analysis (Table 2). Two ACC-HGT showed only one aberration; gain of whole chromosome 16 in case $\mathrm{B}$ and loss of $4 q 13.2-q 22.3$ in case D. Cases E and F harbored 6 and 11 changes, respectively. A detailed description of all copy number changes is given in Table 3 and Fig. 5. Two aberrations were recurrent, loss of 6q23.3-qter and gain of chromosome 8, both in cases $\mathrm{E}$ and $\mathrm{F}$. These two cases also shared two transition points (where a change in the copy number begins or ends), which may indicate a translocation breakpoint. One lies in chromosomal band $6 \mathrm{q} 23.3$ at point 135.7 Mbp, and the second recurrent breakpoint in band 9p22.3 at point 14.1 Mbp (Fig. 4).

The genome-wide profiles of the ACC-HGT differed much from the solid ACC, both in number of alterations (Table 2) and in the specific chromosomes involved in alterations (Fig. 5). The average number of alterations in the 4 ACC-HGT was 4.7 (3 gains and 1.7 loss) whereas the solid ACC demonstrated on average 21.8 events (19 gains and 2.8 losses). The 5 solid 


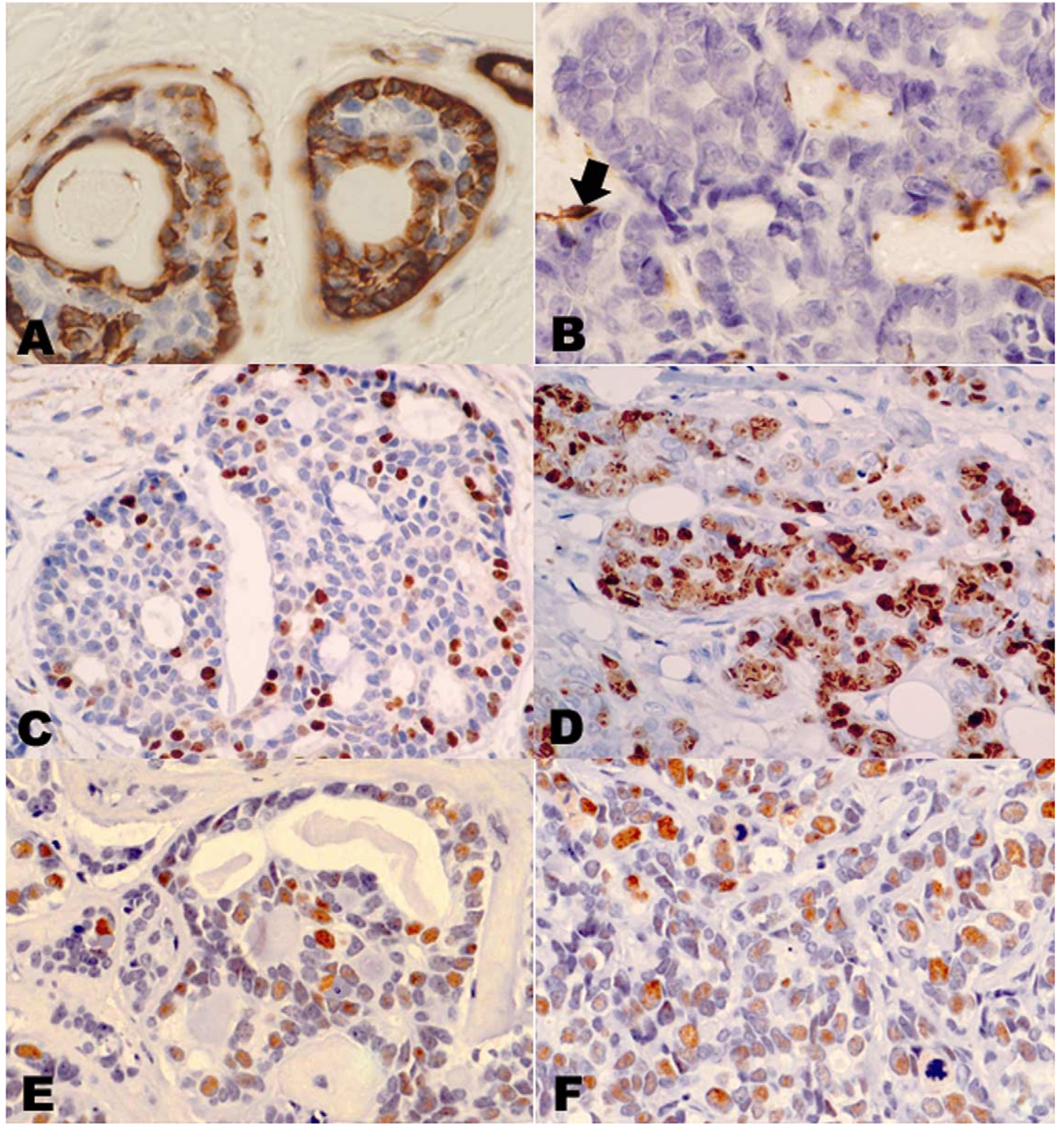

Fig. 2. Expression of $\alpha$-SMA in myoepithelial cells of conventional area (A) and transformed area (B) of ACC-HGT (case F). The transformed component shows few positive myoepithelial cells (arrows) for $\alpha$-SMA, demonstrating the loss of biphasic ductal-myoepithelial differentiation in this area. Ki-67 (case C) and p53 (case A) expression in conventional areas (C, E) and in transformed component (D, F). Original magnification $400 \times(\mathrm{A}, \mathrm{B}, \mathrm{C}$ and D). (Colors are visible in the online version of the article; http://dx.doi.org/10.3233/ACP-CLO-2010-0547.)

ACC showed many recurrent events, of which the most striking were gains at 9q33-q34, 11q13, 11q25, 12q13, $16 \mathrm{p} 13,16 \mathrm{q} 24,19$ and 22 and loss at 14q. Only few of the recurrent aberrations in solid ACC were also seen in the ACC-HGT (Fig. 5).

\subsection{Immunohistochemistry}

All ACC-HGT showed at least focal loss or absence of $\alpha$-SMA and p63 [1] immunoreactivity for myoepithelial cells at the periphery of tumor nests in the transformed component, demonstrating the loss of biphasic ductal-myoepithelial differentiation in this area (Fig. 2).

The Ki-67 index showed a trend for higher expression in the transformed component (both MDA and PDC) compared to the conventional areas (mean 19.6 versus 34.2, $p=0.093$ ) (Fig. 2 and Table 2). Among the ACC-HGT group, a correlation was found between 


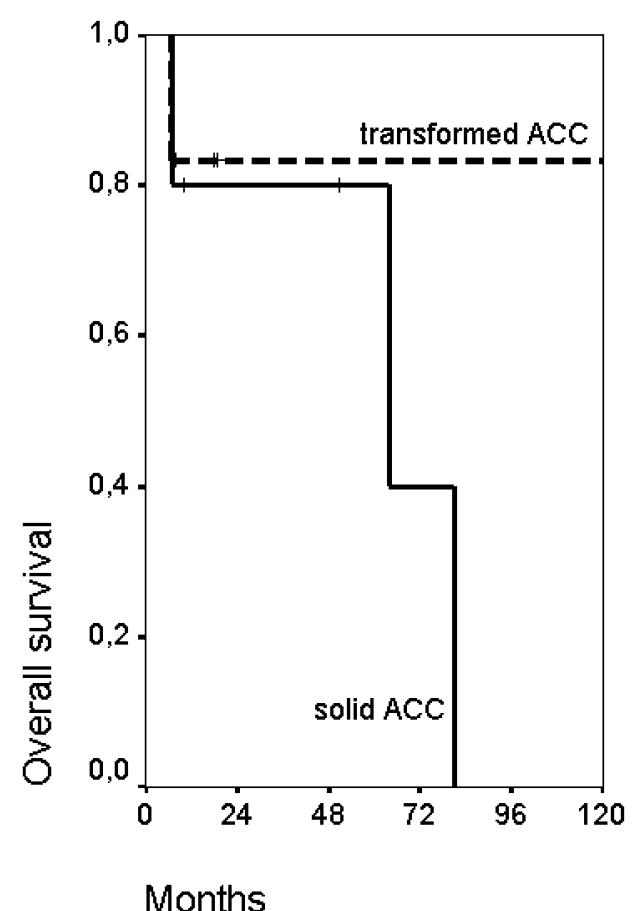

(a)

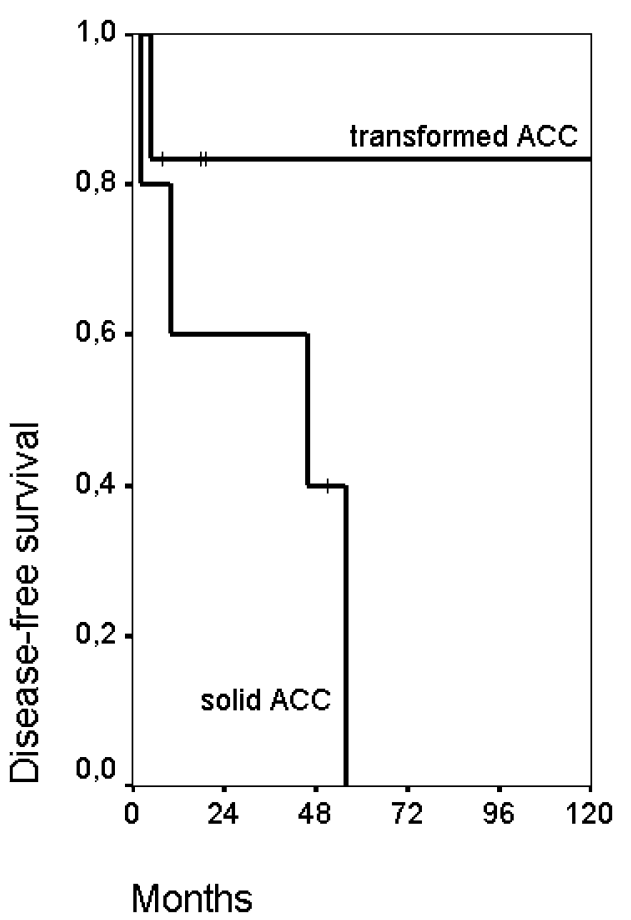

(b)

Fig. 3. (a) Kaplan-Meier curve showing the overall survival of 6 cases ACC-HGT versus 5 cases solid ACC. (b) Kaplan-Meier curve showing the disease-free survival of 6 cases ACC-HGT versus 5 cases solid ACC.

Table 2

Summary of microarray CGH and Ki-67 index

\begin{tabular}{|c|c|c|c|c|c|c|c|}
\hline \multirow[t]{2}{*}{ Cases } & \multicolumn{3}{|c|}{ CNA } & \multicolumn{2}{|c|}{ Ki-67 } & \multicolumn{2}{|c|}{ p53 } \\
\hline & Total & Gains & Losses & $\mathrm{CA}$ & TA & $\mathrm{CA}$ & TA \\
\hline \multicolumn{8}{|c|}{ ACC with transformation } \\
\hline A & - & - & - & 13.7 & 12.5 & 42.5 & 63.1 \\
\hline B & 1 & 1 & 0 & 8.5 & 20.8 & 34.0 & 63.5 \\
\hline $\mathrm{C}$ & - & - & - & 29.0 & 32.0 & 39.6 & 62.5 \\
\hline $\mathrm{D}$ & 1 & 0 & 1 & 27.9 & 33.6 & 34.3 & 64.5 \\
\hline MDA mean & 1.0 & 1.0 & 1.0 & 19.8 & 24.7 & 37.6 & 63.4 \\
\hline $\mathrm{E}$ & 6 & 4 & 2 & 25.5 & 59.9 & 50.2 & 67.7 \\
\hline $\mathrm{F}$ & 11 & 7 & 4 & 13.0 & 46.7 & 33.1 & 56.3 \\
\hline PDC mean & 8.5 & 5.5 & 3.0 & 19.3 & 53.3 & 41.7 & 62.0 \\
\hline Total mean & 4.7 & 3.1 & 1.7 & 19.6 & 34.2 & 38.9 & 62.9 \\
\hline \multicolumn{8}{|l|}{ Solid ACC } \\
\hline 1 & 34 & 31 & 3 & 45.0 & - & 75.0 & - \\
\hline 2 & 25 & 23 & 2 & 5.0 & - & 65.0 & - \\
\hline 3 & 27 & 24 & 3 & 35.0 & - & 80.0 & - \\
\hline 4 & 16 & 11 & 5 & 60.0 & - & 15.0 & - \\
\hline 5 & 7 & 6 & 1 & 20.0 & - & 85.0 & - \\
\hline Total mean & 21.8 & 19 & 2.8 & 33.0 & - & 64.0 & - \\
\hline
\end{tabular}

Notes: CNA - copy number alterations detected by microarray CGH; $\mathrm{CA}$ - conventional area; TA - transformed area. the degree of differentiation (Table 1) of the transformed component and the Ki-67 index. The proliferation index was significantly lower in the MDA than in the PDC group (mean 24.7 versus 53.3, $p=0.028$ ). A comparison of the transformed component of ACCHGT with solid ACC, showed no significant difference in $\mathrm{Ki}-67$ index (mean 34.2 versus 33.0, respectively; $p=0.917$ ). When comparing the solid ACC to MDA and PDC group separately, no significant differences were found in $\mathrm{Ki}-67$ index (mean solid 33.0 versus MDA 24.7 and PDC 53.3; $p=0.502$ and $p=0.270$ ). PDC did show a trend for a higher index but unfortunately there were only two cases in the series.

Neither did we find a significant difference between the solid conventional area of ACC-HGT (cases B$\mathrm{F}$, regardless of the degree of differentiation MDA or PDC) and the solid ACC (mean 20.7 versus 33.0; $p=0.276)$.

In all cases, both components of ACC-HGT showed positive p53 staining (Fig. 2 and Table 2). The p53 expression was significantly higher in the transformed component (both MDA and PDC) than in the conventional area (mean 62.9 versus 38.9, $p=0.000$ ), for group. No significant difference was found between the groups MDA and PDC (mean 63.4 ver- 
Table 3

Detailed description of all gains and losses in 4 transformed ACC

\begin{tabular}{|c|c|c|c|c|c|c|}
\hline Case & Alteration & $\begin{array}{c}\text { Chromosomal } \\
\text { band }\end{array}$ & $\begin{array}{l}\text { Begin } \\
(\mathrm{kbp})\end{array}$ & $\begin{array}{l}\text { End } \\
(\mathrm{kbp})\end{array}$ & $\begin{array}{l}\text { Size } \\
(\mathrm{kbp})\end{array}$ & $\begin{array}{c}\text { Candidate } \\
\text { genes }\end{array}$ \\
\hline B & Gain & 16pter-qter & 0 & 88,669 & 88,669 & Many \\
\hline $\mathrm{D}$ & Deletion & $04 q 13.2-q 22.3$ & 67,364 & 98,431 & 31,067 & Many \\
\hline \multirow[t]{6}{*}{$\mathrm{E}$} & Deletion & 06q23.3-qter & 135,698 & 170,753 & 35,055 & AHI1 \\
\hline & & $12 q 13.11$ & 47,175 & 52,417 & 5242 & Many \\
\hline & Gain & 08pter-qter & 0 & 146,272 & 146,272 & Many \\
\hline & & 09pter-p22.3 & 0 & 14,402 & 14,402 & NFIB \\
\hline & & $10 \mathrm{q} 26.13$ & 123,228 & 123,254 & 26 & FGFR2 \\
\hline & & $11 \mathrm{q} 21$ & 95,558 & 95,715 & 157 & MAML2 \\
\hline \multirow[t]{11}{*}{$\mathrm{F}$} & Deletion & 06q23.3-qter & 135,698 & 170,753 & 35,055 & AHI1 \\
\hline & & 09pter-p22.3 & 0 & 14,119 & 14,119 & NFIB \\
\hline & & 09p22.3-p22.2 & 14,119 & 18,476 & 4357 & Many \\
\hline & & 09p21.3-p13.1 & 21,574 & 38,514 & 16,940 & Many \\
\hline & Gain & 07pter-qter & 0 & 158,821 & 158,821 & Many \\
\hline & & 08pter-qter & 0 & 146,272 & 146,272 & Many \\
\hline & & 06 pter-q23.3 & 0 & 135,698 & 135,698 & AHI1 \\
\hline & & $10 \mathrm{q} 11.21-\mathrm{q} 11.22$ & 45,492 & 47,944 & 2452 & PTPN20A \\
\hline & & 18pter-qter & 0 & 76,116 & 76,116 & Many \\
\hline & & 19pter-qter & 0 & 63,789 & 63,789 & Many \\
\hline & & 20pter-qter & 0 & 62,432 & 62,432 & Many \\
\hline
\end{tabular}

Note: * Candidate genes were only given when the gain or loss, or the breakpoint of a gain or loss, concerned one unique gene.

sus $62.0, p=0.713$ ). Similar expression of $\mathrm{p} 53$ without significant differences was observed between ACC-HGT and solid ACC (mean 62.9 versus 64.0, $p=0.929)$ and also between MDA or PDC group and solid ACC (mean 63.4 (MDA) and 62.0 (PDC) versus $64.0 ; p=0.968$ and $p=0.929$, respectively). The solid conventional areas (cases B-F) of the ACCHGT group showed a trend towards lower p53 expression compared to the solid ACC group (mean 38.2 versus $64.0 ; p=0.085)$.

\subsection{Clinicopathologic-genetic correlations}

The 2 cases of ACC-HGT with transformation into MDA (cases B and D) showed the lowest number of copy number abnormalities and one of the patients was a long-term survivor, who did not develop recurrence or metastasis. Conversely, the solid ACC group and ACC-HGT with transformation into PDC carried the highest number of abnormalities and had the worst clinical course; 3 out of 5 patients with solid type of ACC and one out of two with PDC died of disease (Tables 1 and 2).

\section{Discussion}

An important point of interest with ACC-HGT lies in their proposed poor prognosis, which is suggested to be comparable to or even worse than solid ACC. The median survival of the largest reported series of ACCHGT, in which all cases were poorly differentiated carcinomas, was estimated at 12 months [26], while in solid ACC this is approximately 36-48 months [31]. In addition to recurrence and distant metastasis, a high propensity for lymph node metastasis has been observed, which would indicate a role for neck dissection in these patients [26]. However, ACC-HGT may encompass a wide spectrum of tumors in morphological appearance and probably in biological behavior as well [1].

To date, genetic studies on ACC-HGT have almost exclusively been restricted to protein expression studies with immunohistochemistry $[1,3,4,12,15$, $17,25,26]$. Here, we applied a high-resolution microarray $\mathrm{CGH}$ analysis in an attempt to uncover genes involved in high-grade transformation of ACC, supplemented by immunohistochemical analysis of Ki-67 and p53 and clinico-pathological data. In addition, we 

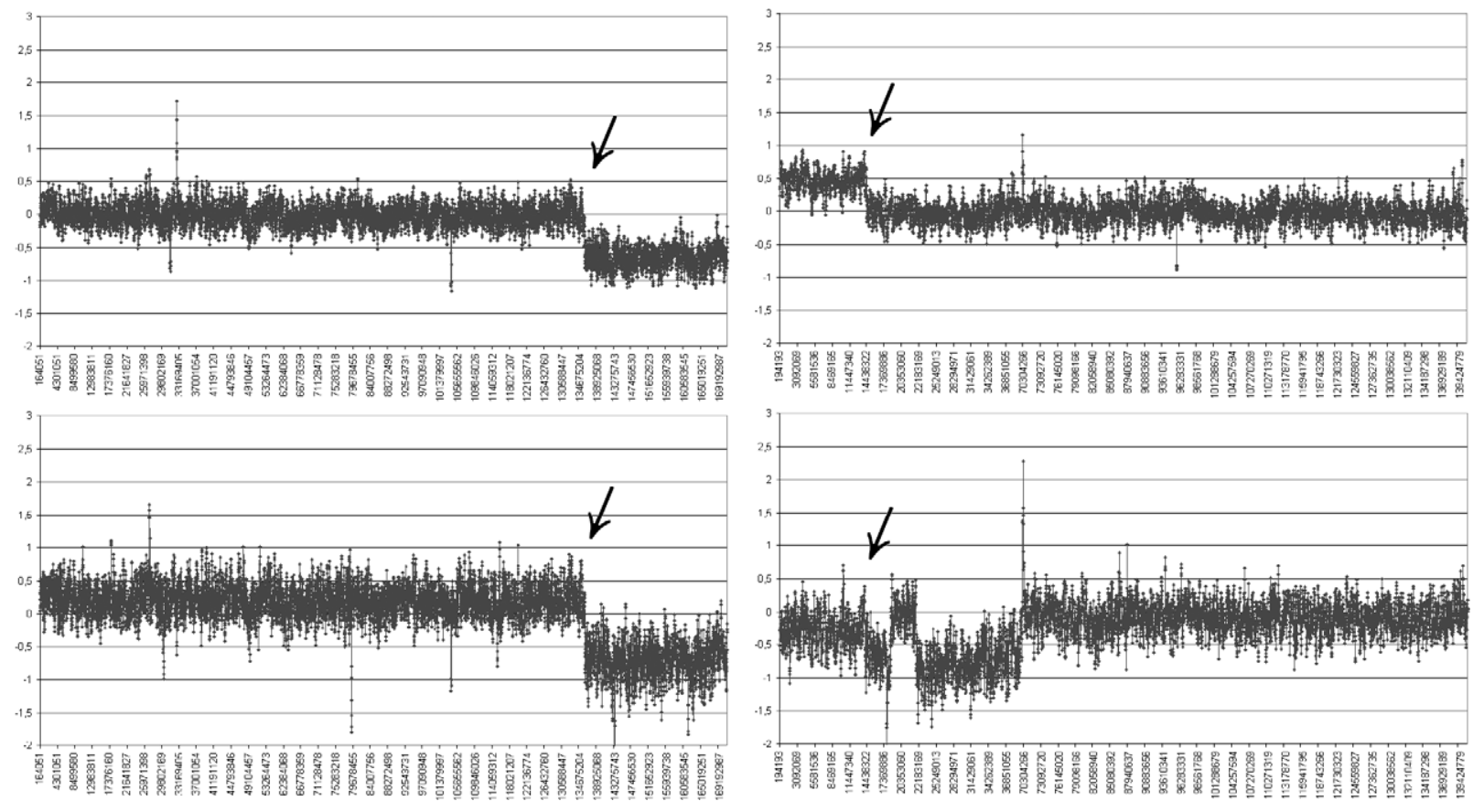

Fig. 4. The left panel shows the microarray CGH profile of chromosome 6 of cases $\mathrm{E}$ and F, both carrying a telomeric deletion that begins at the same 6q23.3 breakpoint, marked by an arrow. The right panel shows the microarray CGH profile of chromosome 9 of cases E, carrying a gain and of case F carrying three distinct regions with copy number loss. The arrows mark the common 9p22.3 breakpoint.

contrasted our data to an existing set of microarray CGH data on solid ACC [32].

An interesting finding in our study was the correlation between the number of chromosomal aberrations and the degree of gland differentiation of the transformed component in the ACC-HGT group. The two MDA had relatively simple genomic profiles carrying one single abnormality, whereas the two PDC showed a higher number of alterations. Solid ACC exhibited even higher numbers of chromosomal aberrations.

ACC with many chromosomal aberrations have been reported to be more aggressive and associated with less favorable outcome than those with few alterations [32]. Also in our series of ACC-HGT the complexity of the genomic profile grossly concurred with the clinical course of the patient: case F with transformation into PDC showed the worst clinical course and the highest number of chromosomal abnormalities. This patient developed lymph node and distant metastasis and died of disease 7 months after diagnosis. In contrast, case B (a MDA with a single chromosomal aberration) was a long-term survivor and did not present metastasis or local recurrence. However, owing to our small number of cases, this association between degree of gland differentiation of the transformed component, amount of chromosomal aberrations and clini- cal outcome needs further confirmation in a larger series.

Two recurrent chromosomal changes were found: deletion at 6q23.3-qter and gain of whole chromosome 8 , both in the two cases of PDC. Both aberrations have been found previously by cytogenetic and LOH analyses in salivary gland tumors [11,14,18,22, $30]$ and in ACC [7,23,24,29]. Rao and collaborators [23] reported that gain of chromosome 8 was significantly associated with ACC solid type. In our series, in the ACC-HGT with PDC, the conventional component was of the solid type. Therefore, we believe that this chromosomal aberration in PDC areas is residual of the parent ACC. In our solid ACC group four cases out of 5 also showed gains of chromosome 8 reinforcing its association with this subtype of ACC.

In the two PDC cases, especially interesting were breaks found at the $6 \mathrm{q}$ and $9 \mathrm{p}$ regions, because they both occurred at exactly the same localization, which could indicate unbalanced chromosomal translocations. Both 6q23 and 9p22 have previously been found involved in translocations in salivary gland tumors $[9,11,14,18,22,30]$ and in ACC [7,23,24,29]. Interestingly, recurrent translocations between $6 \mathrm{q} 23$ and 9p13-23 have been identified before in ACC [11,30] and very recently, Persson et al. [20] identified the 

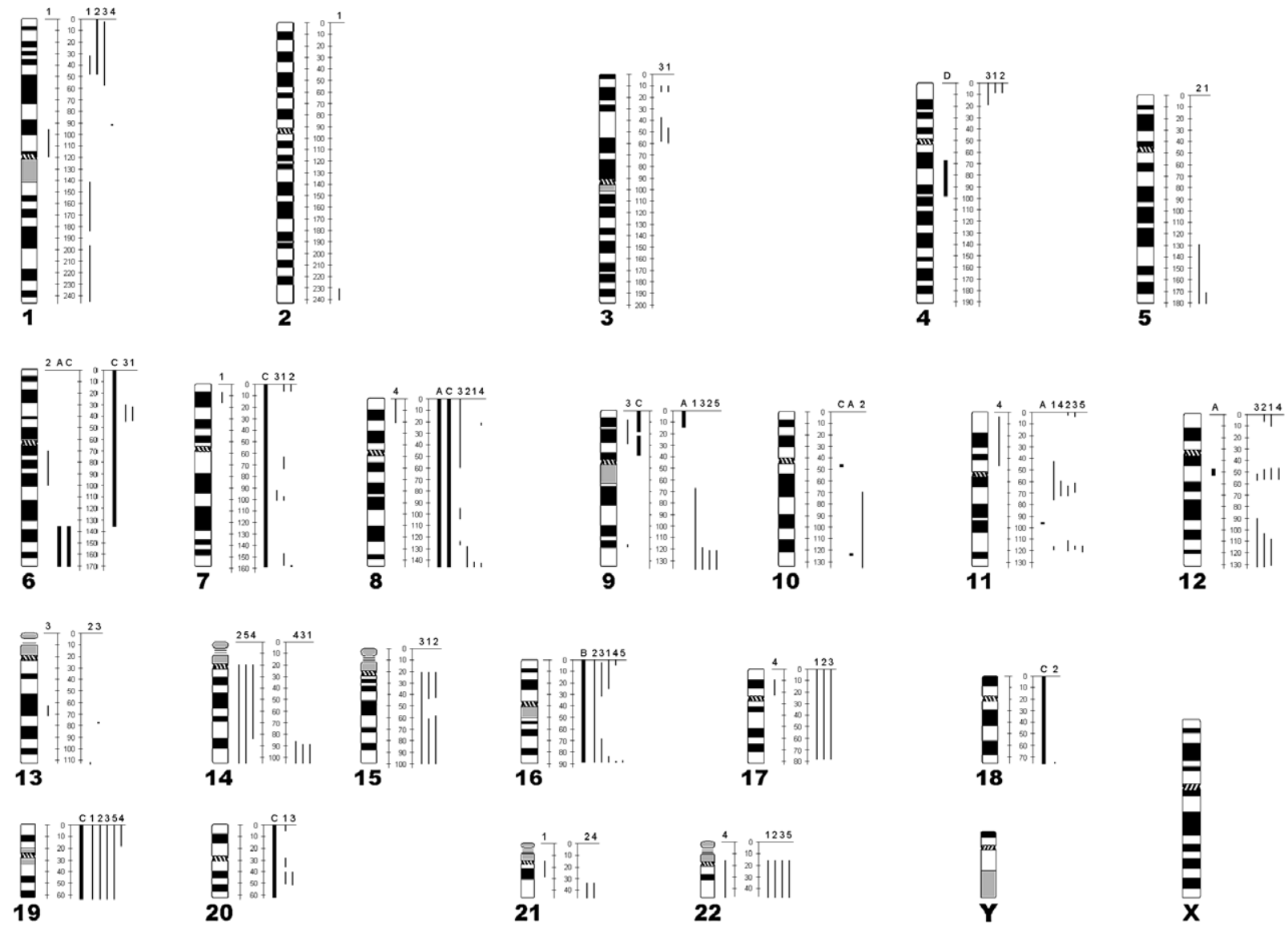

Fig. 5. Overview of all copy number alterations of 4 ACC-HGT (cases B, D, E and F) and 5 solid ACC (cases 1-5). To the right of the pictogram of each chromosome, a scale is placed expressing the number of megabasepairs (Mpb) counting from pter to qter. Copy number losses are presented as bars left to the Mbp-scale and copy number gains to the right.

genes MYB at $6 \mathrm{q} 23$ and NFIB at 9p22 being the fusion partners of this translocation, leading to chimeric transcripts predominantly consisting of MYB and overexpression of MYB protein [20]. In our two cases, the two breaks at 9p22 were located within NFIB in one, and just $100 \mathrm{~kb}$ centromeric to NFIB in the other case. At 6q23.3-qter the two breakpoints were identical, lying within the gene AHI1, which is a close neighbour telomeric of cMYB. Hence it remains unclear if our results confirm the findings of Persson et al. [20]. Overexpression of the oncogene AHI-1 has been implicated in the tumorigenesis of cutaneous T-cell lymphoma [13] and chronic myeloid leukemia [35]. However, we found no studies on a role for AHI-1 in solid tumors.

Although TP53 mutations and/or loss of heterozygosity at the TP53 locus have been suggested to play a role in the pathogenesis of high-grade transformation of ACC $[3,4]$, in the current series none of the cases of ACC with transformation showed chromoso- mal aberration at $17 \mathrm{p} 13$, the locus of TP53. However, positive immunostaining is indicative of mutations in TP53 [27] and all cases in the current series showed positive p53 protein immunoexpression, increasing in the transformed component, suggesting a pivotal role of TP53 in the transformation of ACC.

Finally, our findings do not lend support to the hypothesis that ACC-HGT as a single group or separately (MDA and PDC) is more aggressive than solid ACC [26]. Due to the low number of cases, we cannot conclude whether PDC are more aggressive than MDA. Our data do suggest that the clinical course in ACC-HGT is dependent of the amount of chromosomal abnormalities, in which the poorly differentiated forms (PDC) presents a genetic complexity similar to the solid ACC. Although our series of cases is too small for strong conclusions, we do believe our data are valid, because we found agreement between the genetic, morphology, proliferation index and clin- 
ical data. Perhaps ACC-HGT does not necessarily reflect a more advanced stage of tumor progression, but rather a transformation to another histological form, which encompasses a wide spectrum of carcinomas in terms of aggressiveness [1]. Therefore, the term 'high-grade transformation' may not be adequate for cases of ACC with transformation into MDA. In addition, we propose that for prognostication of ACCHGT, histopathological classification may be supplemented by genomic profiling by microarray CGH copy number analysis or other genome-wide analysis techniques.

\section{Acknowledgements}

This work was financially supported by project EMER 07-048 and PI08-153 of Fondos de Investigación Sanitaria (FIS) and RD06/0020/0034 of Red Temática de Investigación Cooperativa en Cáncer (RTICC), Spain. Supported in Brazil by Fundação de Amparo à Pesquisa do Estado de São Paulo (FAPESP), Grant No. 09/54377-2; Programa de Mobilidade Internacional do Banco Santander/ Universidade Estadual de Campinas (UNICAMP) and Conselho Nacional de Desenvolvimento Científico e Tecnológico (CNPq), Grant No. 473641/2008-9. Special thanks to Bauke Y1stra of Microarray Laboratory of VU University Medical Center, Amsterdam, The Netherlands.

\section{References}

[1] V.L. Bonfitto, A.P. Demasi, A.F. Costa et al., High-grade transformation of adenoid cystic carcinomas may result in adenocarcinomas with wide spectrum of differentiation. A study of the expression of GLUT1 glucose transporter and of mitochondrial antigen in the transformed component, J. Clin. Pathol. 63 (2010), 615-619.

[2] T.E. Buffart, D. Israeli, M. Tijssen et al., Across array comparative genomic hybridization: a strategy to reduce reference channel hybridizations, Genes Chromosomes Cancer 47 (2008), 994-1004.

[3] Y. Chau, T. Hongyo, K. Aozasa et al., Dedifferentiation of adenoid cystic carcinoma: report of a case implicating p53 gene mutation, Hum. Pathol. 32 (2001), 1403-1407.

[4] W. Cheuk, J.K. Chan and R.K. Ngan, Dedifferentiation in adenoid cystic carcinoma of salivary gland: an uncommon complication associated with an accelerated clinical course, Am. J. Surg. Pathol. 23 (1999), 465-472.

[5] D.E. da Cruz Perez, F. de Abreu Alves, I. Nobuko Nishimoto et al., Prognostic factors in head and neck adenoid cystic carcinoma, Oral Oncol. 42 (2005), 139-146.
[6] A.K. El-Naggar and A.G. Huvos, Adenoid cystic carcinoma, in: World Organization Classification of Tumors. Pathology \& Genetics Head and Neck Tumours, L. Barnes, J.W. Everson, P. Reichart et al., eds, IARC Press, Lyon, 2005, pp. 221-222.

[7] W. El-Rifai, S. Rutherford, S. Knuutila et al., Novel DNA copy number losses in chromosome 12q12-q13 in adenoid cystic carcinoma, Neoplasia 3 (2001), 173-178.

[8] G. Franzén, S. Nordgård, M. Boysen et al., DNA content in adenoid cystic carcinomas, Head Neck 17 (1995), 49-55.

[9] J.M. Geurts, E.F. Schoenmakers, E. Röijer et al., Identification of NFIB as recurrent translocation partner gene of HMGIC in pleomorphic adenomas, Oncogene 16 (1998), 865-872.

[10] A. Handra-Luca, D. Planchard and P. Fouret, Docetaxelcisplatin-radiotherapy in adenoid cystic carcinoma with highgrade transformation, Oral Oncol. 45 (2009), 208-209.

[11] K. Higashi, Y. Jin, M. Johansson et al., Rearrangement of 9p13 as the primary chromosomal aberration in adenoid cystic carcinoma of the respiratory tract, Genes Chromosomes Cancer 3 (1991), 21-23.

[12] F. Ide, K. Mishima and I. Saito, Small foci of high-grade carcinoma cells in adenoid cystic carcinoma represent an incipient phase of dedifferentiation, Histopathology 43 (2003), 604606.

[13] E. Kennah, A. Ringrose, L.L. Zhou et al., Identification of tyrosine kinase, $\mathrm{HCK}$, and tumor suppressor, BIN1, as potential mediators of AHI-1 oncogene in primary and transformed CTCL cells, Blood 113 (2009), 4646-4655.

[14] M. Kishi, M. Nakamura, M. Nishimine et al., Loss of heterozygosity on chromosome $6 \mathrm{q}$ correlates with decreased thrombospondin-2 expression in human salivary gland carcinomas, Cancer Sci. 94 (2003), 530-535.

[15] K.P. Malhotra, V. Agrawal and R. Pandey, High grade transformation in adenoid cystic carcinoma of the parotid: report of a case with cytologic, histologic and immunohistochemical study, Head Neck Pathol. 3 (2009), 310-314.

[16] M.A. Moles, I.R. Avila and A.R. Archilla, Dedifferentiation occurring in adenoid cystic carcinoma of the tongue, Oral Surg. Oral Med. Oral Pathol. Oral Radiol. Endod. 88 (1999), 177-180.

[17] T. Nagao, T.A. Gaffey, H. Serizawa et al., Dedifferentiated adenoid cystic carcinoma: a clinicopathologic study of 6 cases, Mod. Pathol. 16 (2003), 1265-1272.

[18] A. Nordkvist, J. Mark, H. Gustafsson et al., Non-random chromosome rearrangements in adenoid cystic carcinoma of the salivary glands, Genes Chromosomes Cancer 10 (1994), 115121.

[19] H. Papadaki, S.D. Finkelstein, S. Kounelis et al., The role of p53 mutation and protein expression in primary and recurrent adenoid cystic carcinoma, Hum. Pathol. 27 (1996), 567-572.

[20] M. Persson, Y. Andrén, J. Mark et al., Recurrent fusion of MYB and NFIB transcription factor genes in carcinomas of the breast and head and neck, Proc. Natl. Acad. Sci. USA 106 (2009), 18740-18744.

[21] K.H. Perzin, P. Gullane and A.C. Clairmont, Adenoid cystic carcinomas arising in salivary glands: a correlation of histologic features and clinical course, Cancer 42 (1978), 265-282.

[22] L. Queimado, A. Reis, I. Fonseca et al., A refined localization of two deleted regions in chromosome $6 \mathrm{q}$ associated with salivary gland carcinomas, Oncogene 16 (1998), 83-88.

[23] R.H. Rao, D. Roberts, Y.J. Zhao et al., Deletion of 1p32-p36 is the most frequent genetic change and poor prognostic marker 
in adenoid cystic carcinoma of the salivary glands, Clin. Cancer Res. 14 (2008), 5181-5187.

[24] S. Rutherford, Y. Yu, C.A. Rumpel et al., Chromosome 6 deletion and candidate tumor suppressor genes in adenoid cystic carcinoma, Cancer Lett. 236 (2006), 309-317.

[25] K. Sato, Y. Ueda, A. Sakurai et al., Adenoid cystic carcinoma of the maxillary sinus with gradual histologic transformation to high-grade adenocarcinoma: a comparative report with dedifferentiated carcinoma, Virchows Arch. 448 (2006), 204-208.

[26] R.R. Seethala, J.L. Hunt, Z.W. Baloch et al., Adenoid cystic carcinoma with high-grade transformation: a report of 11 cases and a review of the literature, Am. J. Surg. Pathol. 31 (2007), 1683-1694.

[27] T. Soussi, The p53 tumor suppressor gene: from molecular biology to clinical investigation, Ann. N. Y. Acad. Sci. 910 (2000), 121-137.

[28] T. Soussi, C. Ishioka, M. Claustres and C. Beroud, Locus specific mutation databases: Pitfalls and good practice based on the p53 experience, Nat. Rev. Cancer 6 (2006), 83-90.

[29] I. Stallmach, P. Zenklusen, P. Komminoth et al., Loss of heterozygosity at chromosome 6q23-25 correlates with clinical and histologic parameters in salivary gland adenoid cystic carcinoma, Virchows Arch. 440 (2002), 77-84.
[30] G. Stenman, J. Sandros, R. Dahlenfors et al., 6q- and loss of the Y chromosome - two common deviations in malignant human salivary gland tumors, Cancer Genet. Cytogenet. 22 (1986), 283-293.

[31] P.A. Szanto, M.A. Luna, M.E. Tortoledo et al., Histologic grading of adenoid cystic carcinoma of the salivary glands, Cancer 54 (1984), 1062-1069.

[32] H. Vékony, B. Ylstra, S.M. Wilting et al., DNA copy number gains at loci of growth factors and their receptors in salivary gland adenoid cystic carcinoma, Clin. Cancer Res. 13 (2007), 3133-3139.

[33] L.E. Vissers, J.A. Veltman, A.G. van Kessel and H.G. Brunner, Identification of disease genes by whole genome CGH arrays, Hum. Mol. Genet. 14 (2005), 215-223.

[34] B.M. Wening, Neoplasmas of the salivary glands, in: Atlas of Head and Neck Pathology, B.M. Wening and C.S. Heffess, eds, Saunders Elsevier, China, 2008, pp. 628-631.

[35] L.L. Zhou, Y. Zhao, A. Ringrose et al., AHI-1 interacts with BCR-ABL and modulates BCR-ABL transforming activity and imatinib response of CML stem/progenitor cells, J. Exp. Med. 205 (2008), 2657-2671. 


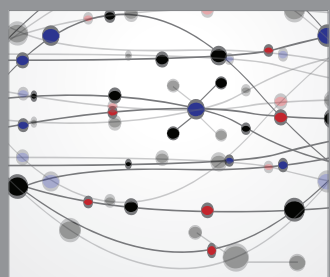

The Scientific World Journal
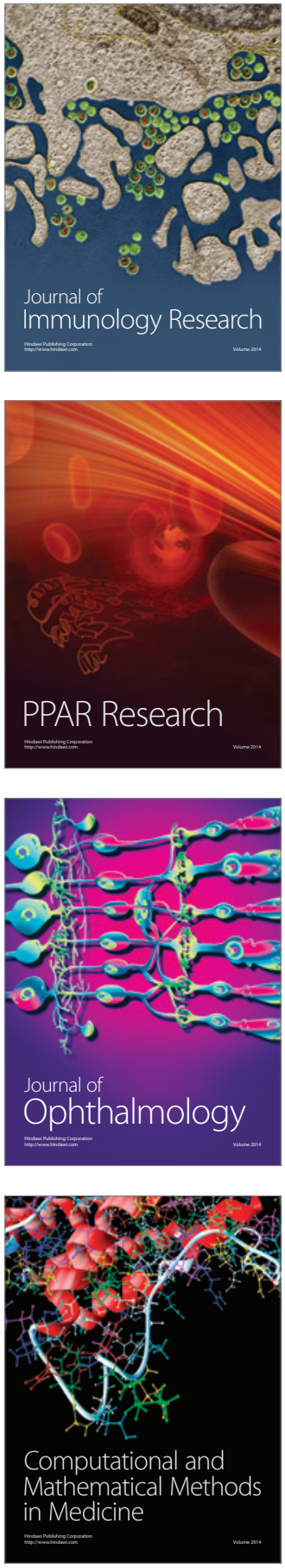

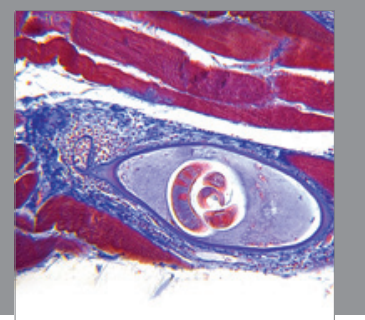

Gastroenterology

Research and Practice
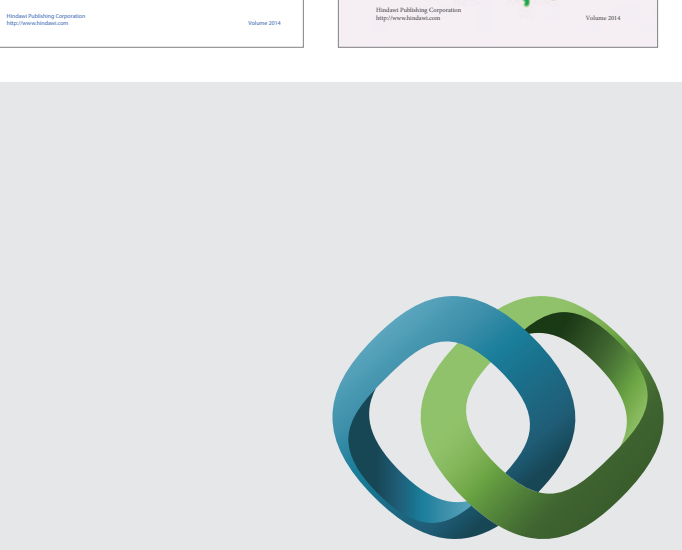

\section{Hindawi}

Submit your manuscripts at

http://www.hindawi.com
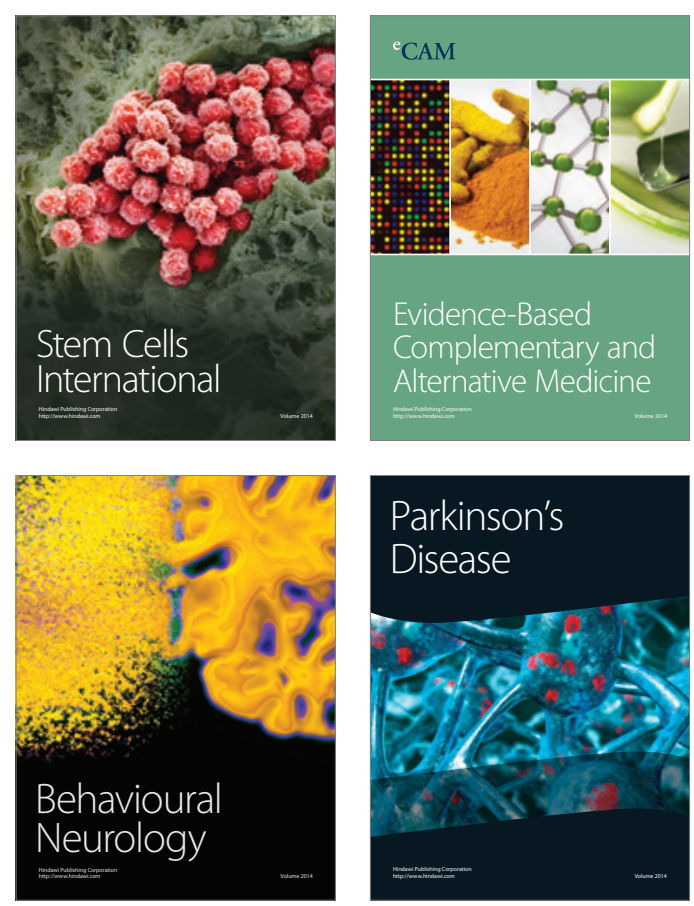

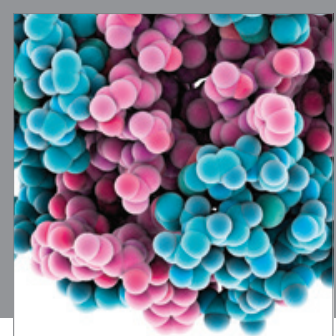

Journal of
Diabetes Research

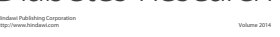

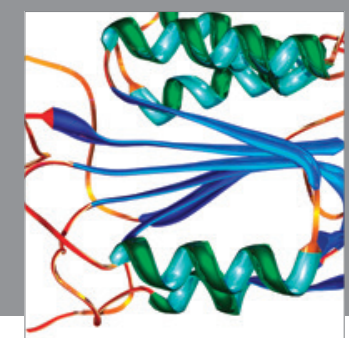

Disease Markers
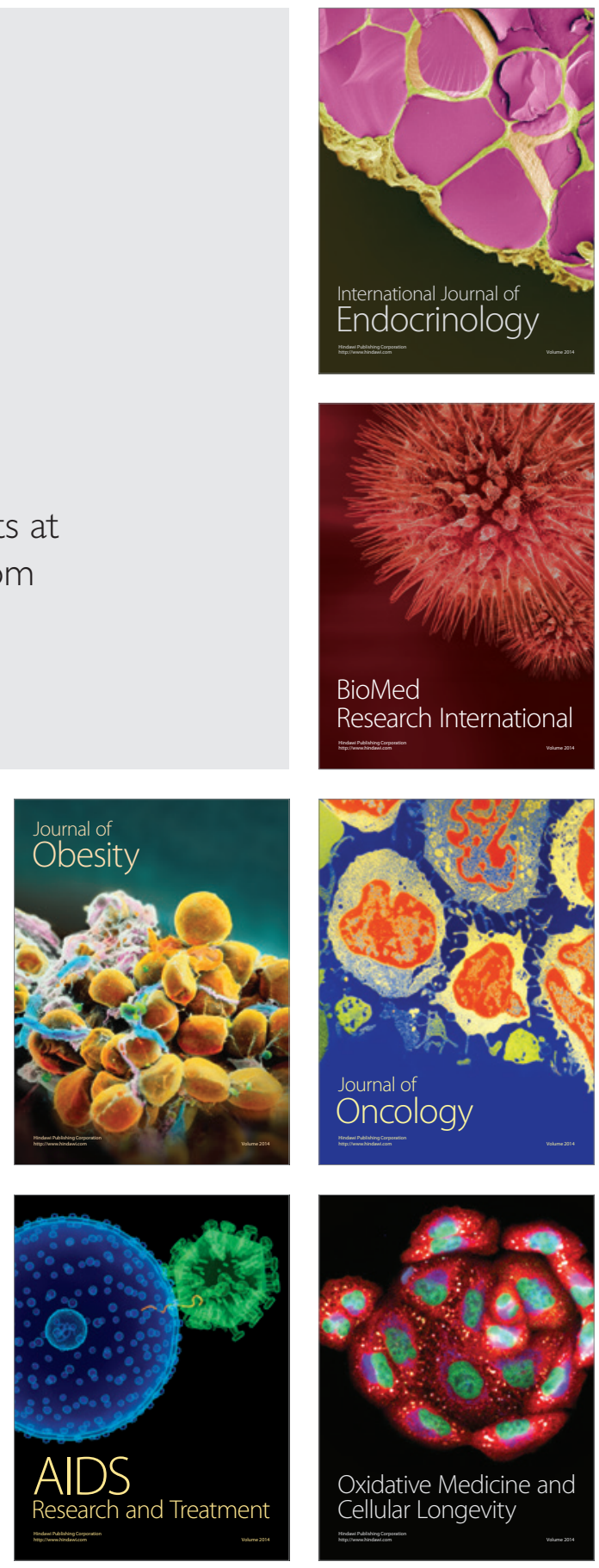